A C G Rec. Nat. Prod. 13:1 (2019) 96-96

publications

records of natural products

\title{
ERRATUM
}

\section{Erratum to "Headspace Analysis of Volatile Compounds Coupled to Chemometrics in Leaves from the Magnoliaceae Family"} [Rec. Nat. Prod. 9:1 (2015) 153-158]

\author{
Mohamed A. Farag ${ }^{1,2 *}$, Riham Salah EI Din ${ }^{1}$ and Sherifa Fahmy ${ }^{1}$ \\ ${ }^{1}$ Pharmacognosy Department, College of Pharmacy, Kasr El-Aini St, Cairo University, Egypt \\ P.B.11562. \\ ${ }^{2}$ Center for Regulatory and Environmental Analytical Metabolomics, Department of Chemistry, \\ University of Louisville 2320 S. Brook St., Louisville, Egypt KY 40292
}

In the published paper affiliations for coauthors Riham S. El Din \& and Sherifa Fahmy is given a wrong number $\mathbf{2}$ and to be thus affiliated to Center for Regulatory and Environmental Analytical Metabolomics, Department of Chemistry, University of Louisville 2320 S. Brook St., Louisville and should be 1 to Pharmacognosy Department, College of Pharmacy, Kasr El-Aini St, Cairo University, Egypt. The authors apologize for this error and their affiliation number changed as $\mathbf{1}$. 